ULB-TH/04-08

gr-qc/0404006

\title{
Classification of surface charges for a spin 2 field on a curved background solution
}

\author{
Glenn Barnich* \\ Physique Théorique et Mathématique and International Solvay Institutes, \\ Université Libre de Bruxelles, \\ Campus Plaine C.P. 231, B-1050 Bruxelles, Belgique \\ Serge Leclercq and Philippe Spindel \\ Mécanique \& Gravitation, Faculté des Sciences, \\ Université de Mons-Hainaut \\ 20, Place du Parc, B-7000 Mons, Belgique
}

\begin{abstract}
We give an explicit proof of the result that non trivial conserved $n-2$ forms for a spin 2 field on a background corresponding to a solution to Einstein's equation (with or without cosmological constant) are characterized uniquely by the Killing vector fields of the background.
\end{abstract}

* Research Associate of the National Fund for Scientific Research (Belgium). 
In dimensions $n>2$, a consistent theory for a spin 2 field $h_{\mu \nu}$ on an Einstein spacetime background can be constructed by taking the quadratic part of the expansion of the Einstein-Hilbert action,

$$
S[g]=\frac{1}{16 \pi} \int d^{n} x \sqrt{|g|}(R-2 \Lambda),
$$

around a given background solution $\bar{g}_{\mu \nu}$. If $g_{\mu \nu}=\bar{g}_{\mu \nu}+h_{\mu \nu}$,

$$
\begin{array}{r}
S^{(2)}[h ; \bar{g}]=\frac{1}{32 \pi} \int d^{n} x \sqrt{|\bar{g}|}\left(-\frac{1}{2} \bar{D}_{\lambda} h_{\mu \nu} \bar{D}^{\lambda} h^{\mu \nu}+\bar{D}_{\lambda} h_{\mu \nu} \bar{D}^{\mu} h^{\nu \lambda}-\bar{D}_{\nu} h \bar{D}_{\mu} h^{\mu \nu}\right. \\
\left.+\frac{1}{2} \bar{D}^{\lambda} h \bar{D}_{\lambda} h+\frac{\Lambda}{n-2}\left(2 h_{\mu \nu} h^{\mu \nu}-h^{2}\right)\right)
\end{array}
$$

where $\bar{g}_{\mu \nu}$, together with its inverse $\bar{g}^{\mu \nu}$, is used to lower and raise the indices, $h=h_{\mu}^{\mu}$ and $\bar{D}_{\mu}$ denotes the associated covariant derivative. In particular, the diffeomorphism invariance of the Einstein-Hilbert implies the invariance of the action (2) under the gauge transformations

$$
\delta_{\xi} h_{\mu \nu}=L_{\xi} \bar{g}_{\mu \nu}
$$

with arbitrary parameters $\xi^{\mu}(x)$.

An $n-2$ form $k$ that depends on the space-time coordinates $x^{\mu}$, the fields $h_{\mu \nu}^{a}$ and a finite number of their derivatives ${ }^{1}$ is conserved if

$$
d k \approx 0
$$

Here $d$ is the so-called exterior or horizontal differential defined by

$$
d=d x^{\lambda}\left(\frac{\partial}{\partial x^{\lambda}}+h_{\mu \nu, \lambda}^{a} \frac{\partial}{\partial h_{\mu \nu}^{a}}+\ldots\right)
$$

while $\approx$ means an equality that holds "on-shell", i.e., for all solutions of the EulerLagrange equations associated to (2). A conserved $n-2$ form $k$ is defined to be trivial if

$$
k \approx d m \quad
$$

for some $n-3$ form $m$, and two conserved $n-2$ forms are considered equivalent if they differ by a trivial conserved $n-2$ form. By definition, these equivalence classes are elements of $H_{\text {char }}^{n-2}(d)$, the characteristic cohomology of $d$ in form degree $n-2$ (see e.g. [5, 6, 7, 3, 8]), which we consider here as a vector space over $\mathbb{R}$.

Our aim in this note is to compute the cohomology group $H_{\text {char }}^{n-2}(d)$ for the equations of motion associated to the action (2). The result of this computation has been originally presented in 9. However, since the detailed proof is not given and does not seem to be readily available in the literature, we will fill this gap below.

There are several reasons why the group $H_{\text {char }}^{n-2}(d)$ is relevant.

\footnotetext{
${ }^{1}$ All forms that we consider below are supposed to have this kind of dependence. In the context of the variational bicomplex (see e.g. 1, 2, 3, 4, for more details), such forms are called horizontal forms.
} 
- In the context of the spin 2 theory itself, conserved $n-2$ forms give rise, upon integration over closed $n-2$ dimensional surfaces $\mathcal{C}^{n-2}$ and evaluation for a given solution, to charges that depend only on the homology class of $\mathcal{C}^{n-2}$ and the equivalence class of $k$.

- For space-times $g_{\mu \nu}$ that asymptotically near some boundary approach the background metric $\bar{g}_{\mu \nu}$, the appropriate generalization to the asymptotic context of the forms $k$ can be used to define boundary charges that give information on the properties of the space-times in the bulk (see e.g. [10, 9, 11]).

Alternatively, if $\bar{g}_{\mu \nu}$ denotes a given solution, like a Kerr black hole for instance, and $\bar{g}_{\mu \nu}+h_{\mu \nu}$ an infinitesimal perturbation of this solution, then the charges associated to $k$ are the same when evaluated on 2 closed $n-2$-dimensional hypersurfaces that are the boundary of some $n-1$-dimensional hypersurface. This property has been used in [12] by using the 2-sphere at infinity and the bifurcation 2-sphere of a Killing horizon to derive the first law of black hole mechanics.

Recently an improved version of the forms $k$ has been shown to be useful in full general relativity to compute the boundary charges on surfaces in the bulk and to interpolate between solutions that are not infinitesimally close [13.

- For the problem of consistently deforming the theory described by (2) and (3) (or several copies of it), the characteristic cohomology in form degree $n-2$ is related to deformations that modify the algebra of the gauge transformations (3) in a non trivial way. Indeed, a cohomological formulation [14] (see also [15, 16]) of the problem of consistent deformations of gauge theories [17] shows that first order deformations are controlled by the local BRST cohomology group in ghost number 0 . In turn, an important ingredient in the computation of this group is the cohomology group $H_{2}^{n}(\delta \mid d)$ of the Koszul Tate differential $\delta$, associated to the resolution of forms pulled-back to the surface defined by the equations of motion, modulo the horizontal differential $d$. This last group is directly related to non trivial deformations of the gauge algebra and can be shown [18 to be isomorphic to the characteristic cohomology in degree $n-2$.

The deformation problem for a collection of spin 2 fields on a flat background has been considered in details in 19. The results obtained below are a straightforward generalization to the curved case of the results discussed in the proof of theorem 4.2 of [19].

We now prove that the fields $h_{\mu \nu}$ and their derivatives can be split into independent variables $y_{A}$ not constrained by the equations of motion and a set of variables $z^{a}$, in one-to-one correspondence, at each order of the number of derivatives involved, with the independent equations of motion [see [18, section 3]. In finding such a split, one has to bear in mind that due to the Noether identities associated to the gauge transformations (3)

$$
\bar{D}_{\mu} \frac{\delta S^{(2)}}{\delta h_{\mu \nu}} \equiv 0
$$


not all equations of motions are independent. Indeed the equations defined by

$$
\left\{\mathcal{L}_{\Delta}\right\}=\left\{\partial_{0} \frac{\delta S^{(2)}}{\delta h_{0 \nu}}, \partial_{\rho} \partial_{0} \frac{\delta S^{(2)}}{\delta h_{0 \nu}}, \partial_{\rho_{1}} \partial_{\rho_{2}} \partial_{0} \frac{\delta S^{(2)}}{\delta h_{0 \nu}}, \ldots,\right\}
$$

can be considered as dependent equations that hold as a consequence of the remaining equations

$$
\begin{array}{r}
\left\{\mathcal{L}_{a}\right\}=\left\{\frac{\delta S^{(2)}}{\delta h_{\mu \nu}}, \partial_{k} \frac{\delta S^{(2)}}{\delta h_{0 \nu}}, \partial_{k_{1}} \partial_{k_{2}} \frac{\delta S^{(2)}}{\delta h_{0 \nu}}, \ldots,\right. \\
\left.\partial_{\rho} \frac{\delta S^{(2)}}{\delta h_{m n}}, \partial_{\rho_{1}} \partial_{\rho_{2}} \frac{\delta S^{(2)}}{\delta h_{m n}}, \ldots,\right\},
\end{array}
$$

where Greek indices run from 0 to $n-1$, while Latin indices run from 1 to $n-1$. In fact, the equations defined by $\left\{\mathcal{L}_{a}\right\}$ will be shown below to be all independent by solving them for independent variables $\left\{z^{a}\right\}$. At the same time, this allows us to show that the irreducible set of gauge transformations (3) is in fact a generating set.

First we note that the equations of motion can be reexpressed as

$$
\frac{\delta S^{(2)}}{\delta h_{\mu \nu}}=\frac{\sqrt{|g|}}{32 \pi}\left(K^{\mu \nu}-\frac{1}{2} \bar{g}^{\mu \nu} K\right),
$$

where

$$
K^{\mu \nu}=\bar{D}^{\mu} \bar{D}^{\nu} h+\bar{D}_{\lambda} \bar{D}^{\lambda} h^{\mu \nu}-2 \bar{D}_{\lambda} \bar{D}^{(\mu} h^{\nu) \lambda}+\frac{4 \lambda}{n-2} h^{\mu \nu}
$$

and $K=\bar{g}_{\mu \nu} K^{\mu \nu}$. Thus the equations defined by $\delta S^{(2)} / \delta h_{\mu \nu}$ and their derivatives are equivalent to those defined by $K^{\mu \nu}$ and their derivatives. Because the Noether identities in terms of $K^{\mu \nu}$ can still be solved for $\partial_{0} K^{0 \nu}$, there is a similar split into $\left\{K_{\Delta}\right\}$ and $\left\{K_{a}\right\}$ as the one into $\left\{\mathcal{L}_{\Delta}\right\}$ and $\left\{\mathcal{L}_{a}\right\}$. If we assume the diagonal contravariant elements of the metric everywhere non zero ${ }^{2}$, i.e., $\bar{g}^{\mu \mu} \neq 0$ for $\mu=0, \ldots, n-1$, we may introduce variables $z^{a}$ such that $z^{a}=\mathcal{Z}^{a}\left[K_{b}, y_{A}\right]$, where

$$
\left\{z^{a}\right\}=\left\{\begin{array}{c}
\partial_{0} \partial_{0} h_{k l}, \partial_{\rho} \partial_{0} \partial_{0} h_{k l}, \partial_{\rho_{1}} \partial_{\rho_{2}} \partial_{0} \partial_{0} h_{k l}, \ldots, \\
\partial_{1} \partial_{1} h_{0 \bar{\lambda}}, \partial_{k} \partial_{1} \partial_{1} h_{0 \bar{\lambda}}, \partial_{k_{1}} \partial_{k_{2}} \partial_{1} \partial_{1} h_{0 \bar{\lambda}}, \ldots, \\
\left.\partial_{0} \partial_{1} h_{22}, \partial_{k} \partial_{0} \partial_{1} h_{22}, \partial_{k_{1}} \partial_{k_{2}} \partial_{0} \partial_{1} h_{22} \ldots\right\}
\end{array},\right.
$$

with the independent variables, not constrained by the equations of motion, chosen as

$$
\begin{aligned}
& \left\{y_{A}\right\}=\left\{\quad h_{\mu \nu}, \partial_{\rho} h_{\mu \nu},\right. \\
& \partial_{m} \partial_{\rho} h_{k \tilde{l}}, \partial_{m_{1}} \partial_{m_{2}} \partial_{\rho} h_{k \tilde{l}}, \ldots, \\
& \partial_{\rho_{1}} \partial_{\rho_{2}} h_{01}, \partial_{\rho_{1}} \partial_{\rho_{2}} \partial_{\rho_{3}} h_{01}, \ldots, \\
& \partial_{\rho} \partial_{0} h_{0 \bar{\lambda}}, \partial_{\rho_{1}} \partial_{\rho_{2}} \partial_{0} h_{0 \bar{\lambda}}, \ldots, \\
& \partial_{\bar{k}} \partial_{l} h_{0 \bar{\lambda}}, \partial_{\bar{k}_{1}} \partial_{\bar{k}_{2}} \partial_{l} h_{0 \bar{\lambda}}, \ldots, \\
& \partial_{\bar{k}} \partial_{0} h_{22}, \partial_{\bar{k}_{1}} \partial_{\bar{k}_{2}} \partial_{0} h_{22}, \ldots, \\
& \left.\partial_{k_{1}} \partial_{k_{2}} h_{22}, \partial_{k_{1}} \partial_{k_{2}} \partial_{k_{3}} h_{22}, \ldots\right\} .
\end{aligned}
$$

\footnotetext{
${ }^{2}$ This can always be done locally by choosing for instance a normal geodesic coordinate system.
} 
Here barred Greek indices run from 0 to $n-1$ without taking the value 1 and barred Latin indices run from 2 to $n-1$ while tilded Latin indices run from 1 to $n-1$ without taking the value 2 . One can see that the set of $y_{A}$ variables is stable with respect to the $\partial_{\bar{l}}$ derivatives : for any variable $y_{A}$, the derivative $\partial_{\bar{l}} y_{A}$ belongs to the set of $y$-variable. Accordingly the $y_{A}$ variables can be ordered according to their number $m$ of derivatives of type $\partial_{\bar{l}}$. Thus the "Cauchy order" of this perturbation theory is, at most, equal to 2. Let us notice that the same result can be obtained if we assume that globally the background metric verifies the conditions: $\bar{g}^{01} \neq 0$ and $\bar{g}^{\overline{l l}} \neq 0$.

After these preliminary considerations, the first step in the computation of $H_{\text {char }}^{n-2}(d)$ is the characterization of the isomorphic group $H_{2}^{n}(\delta \mid d)$ (see section 6.2 of [20]). For irreducible linear gauge theory, the latter group can be shown to be given by equivalence classes of field dependent gauge parameters such that the corresponding gauge transformations are antisymmetric combinations of the equations of motion, with two parameters being equivalent if they coincide on-shell (see [20] theorem 6.7, which applies not only to normal but also to linear irreducible gauge theories such as the one considered here). Explicitly, we need to characterize the vector space $\operatorname{Span}_{\mathbb{R}}\left\{\left[f_{\mu}\right]\right\}$ defined by

$$
\bar{D}_{\mu} f_{\nu}+\bar{D}_{\nu} f_{\mu} \approx^{a s} 0 \quad, \quad f_{\mu} \sim f_{\mu}+t_{\mu}, t_{\mu} \approx 0
$$

where $f_{\mu}$ depend on $x^{\nu}, h_{\lambda \rho}$ and a finite number of their derivatives while $\approx^{a s}$ denotes antisymmetric combinations of the equations of motion in the sense of (6.25) of [20].

In particular, $f_{\mu}$ satisfies $F_{\mu \nu} \equiv \bar{D}_{\mu} f_{\nu}+\bar{D}_{\nu} f_{\mu} \approx 0$. Applying $\bar{D}_{\lambda}$ one gets $\bar{D}_{\lambda} F_{\mu \nu} \approx 0$. Adding $\bar{D}_{\mu} F_{\lambda \nu} \approx 0$, subtracting $\bar{D}_{\nu} F_{\lambda \mu} \approx 0$, using $\left[\bar{D}_{\alpha}, \bar{D}_{\beta}\right] f_{\gamma}=-\bar{R}_{\alpha \beta \gamma}^{\delta} f_{\delta}$ three times, gives, by using in addition the symmetry properties of the Riemann tensor,

$$
\bar{D}_{\lambda} \bar{D}_{\mu} f_{\nu}+\bar{R}_{\lambda \nu \mu}^{\sigma} f_{\sigma} \approx 0
$$

By using the freedom of adding terms that vanish when the equations of motion hold, one can assume that $f_{\mu}=f_{\mu}(x, y)$, where $f_{\mu}(x, y)$ depends on the $\partial_{\bar{l}}$ derivatives of $y_{A}$ up to some maximum order $M$. By taking $\lambda, \mu \geq 2$ in (10) and considering the dependence of $f_{\nu}$ on $y_{A}$ of maximum order, (10) implies that $f_{\nu}$ cannot depend on the variables $y_{A}$ of order $M$. Hence, by induction, $f_{\nu}$ cannot depend on the variables $y_{A}$ at all, and we have shown that $f_{\mu}=f_{\mu}(x)+t_{\mu}$. Furthermore, $f_{\mu}(x) \approx 0$ implies $f_{\mu}(x)=0$, so that a solution $f_{\mu}(x)$ is trivial if and only if it vanishes. For $f_{\mu}(x)$, the equation $\bar{D}_{\mu} f_{\nu}+\bar{D}_{\nu} f_{\mu} \approx^{a s} 0$ reduces to

$$
\bar{D}_{\mu} f_{\nu}+\bar{D}_{\nu} f_{\mu}=0
$$

which is the Killing equation for $\bar{g}_{\mu \nu}$.

Hence, we have explicitly shown that $H_{\text {char }}^{n-2}(d)$ is isomorphic to the vector space of Killing vectors $\tilde{\xi}^{\mu}$ of $\bar{g}_{\mu \nu}$.

The construction of associated $\left[k_{\tilde{\xi}}\right]$ of $H_{\text {char }}^{n-2}(d)$ is explained from a general point of view in [11], section 3, while section 6.3.1 contains the explicit expressions for the case at hand and recovers those originally obtained in [10, 12, 9]. 


\section{Acknowledgements}

The work of G. B. is supported in part by the "Actions de Recherche Concertées" of the "Direction de la Recherche Scientifique-Communauté Française de Belgique", by a "Pôle d'Attraction Interuniversitaire" (Belgium), by IISN-Belgium, convention 4.4505.86, by the European Commission RTN program HPRN-CT00131, in which the authors are associated to K. U. Leuven and by Proyectos FONDECYT 1970151, 7960001 (Chile).

S. L. and Ph. S. acknowledge support from the Fonds National de la Recherche Scientifique through an F.R.F.C. grant.

\section{References}

[1] P. Olver, Applications of Lie Groups to Differential Equations. Spinger Verlag, New York, 2nd ed., 1993. 1st ed., 1986.

[2] D. J. Saunders, The Geometry of Jet bundles. Cambridge University Press, 1989.

[3] I. Anderson, "Introduction to the variational bicomplex," in Mathematical Aspects of Classical Field Theory, M. Gotay, J. Marsden, and V. Moncrief, eds., vol. 132 of Contemporary Mathematics, pp. 51-73. Amer. Math. Soc., 1992.

[4] I. Anderson, "The variatonal bicomplex," tech. rep., Formal Geometry and Mathematical Physics, Department of Mathematics, Utah State University, 1989.

[5] A. M. Vinogradov, "A spectral sequence associated with a nonlinear differential equation and algebra-geometric foundations of Lagrangian field theory with constraints," Sov. Math. Dokl. 19 (1978) 144.

[6] T. Tsujishita, "On variational bicomplexes associated to differential equations," Osaka J. Math. 19 (1982) 311.

[7] A. M. Vinogradov, "The C-spectral sequence, Lagrangian formalism, and conservation laws. I. The linear theory. II. The non linear theory," J. Math. Anal. Appl. 100 (1984) 1.

[8] R. L. Bryant and P. A. Griffiths, "Characteristic cohomology of differential systems I: General theory," J. Am. Math. Soc. 8 (1995) 507.

[9] I. M. Anderson and C. G. Torre, "Asymptotic conservation laws in field theory," Phys. Rev. Lett. 77 (1996) 4109-4113, hep-th/9608008.

[10] L. F. Abbott and S. Deser, "Stability of gravity with a cosmological constant," Nucl. Phys. B195 (1982) 76.

[11] G. Barnich and F. Brandt, "Covariant theory of asymptotic symmetries, conservation laws and central charges," Nucl. Phys. B633 (2002) 3-82, hep-th/0111246 
[12] V. Iyer and R. M. Wald, "Some properties of Noether charge and a proposal for dynamical black hole entropy," Phys. Rev. D50 (1994) 846-864, gr-qc/9403028.

[13] G. Barnich, "Boundary charges in gauge theories: Using Stokes theorem in the bulk," Class. Quant. Grav. 20 (2003) 3685-3698, hep-th/0301039

[14] G. Barnich and M. Henneaux, "Consistent couplings between fields with a gauge freedom and deformations of the master equation," Phys. Lett. B311 (1993) 123-129, hep-th/9304057.

[15] J. Stasheff, "Deformation theory and the Batalin-Vilkovisky master equation," q-alg/9702012.

[16] M. Henneaux, "Consistent interactions between gauge fields: The cohomological approach," in Secondary Calculus and Cohomological Physics, A. V. M. Henneaux, J. Krasil'shchik, ed., vol. 219 of Contemporary Mathematics, pp. 93-109. Amercian Mathematical Society, 1997. hep-th/9712226.

[17] F. A. Berends, G. J. H. Burgers, and H. van Dam, "On the theoretical problems in constructing interactions involving higher spin massless particles," Nucl. Phys. B260 (1985) 295.

[18] G. Barnich, F. Brandt, and M. Henneaux, "Local BRST cohomology in the antifield formalism. I. General theorems," Commun. Math. Phys. 174 (1995) 57-92, hep-th/9405109

[19] N. Boulanger, T. Damour, L. Gualtieri, and M. Henneaux, "Inconsistency of interacting, multigraviton theories," Nucl. Phys. B597 (2001) 127-171, hep-th/0007220

[20] G. Barnich, F. Brandt, and M. Henneaux, "Local BRST cohomology in gauge theories," Phys. Rept. 338 (2000) 439-569, hep-th/0002245. 